\title{
Ketogenic diet for mitochondrial disease: a systematic review on efficacy and safety
}

\author{
Heidi Zweers ${ }^{1,2^{*}+}$, Annemiek M. J. van Wegberg ${ }^{1,2+}$, Mirian C. H. Janssen ${ }^{2,4}$ and Saskia B. Wortmann ${ }^{2,3}$
}

\begin{abstract}
Background: No curative therapy for mitochondrial disease (MD) exists, prioritizing supportive treatment for symptom relief. In animal and cell models ketones decrease oxidative stress, increase antioxidants and scavenge free radicals, putting ketogenic diets (KDs) on the list of management options for MD. Furthermore, KDs are well-known, safe and effective treatments for epilepsy, a frequent symptom of MD. This systematic review evaluates efficacy and safety of KD for MD.

Methods: We searched Pubmed, Cochrane, Embase and Cinahl (November 2020) with search terms linked to MD and KD. From the identified records, we excluded studies on Pyruvate Dehydrogenase Complex deficiency. From these eligible reports, cases without a genetically confirmed diagnosis and cases without sufficient data on KD and clinical course were excluded. The remaining studies were included in the qualitative analysis.

Results: Only 20 cases ( 14 pediatric) from the 694 papers identified met the inclusion criteria (one controlled trial $(n=5), 15$ case reports). KD led to seizure control in 7 out of 8 cases and improved muscular symptoms in 3 of 10 individuals. In 4 of 20 cases KD reversed the clinical phenotype (e.g. cardiomyopathy, movement disorder). In 5 adults with mitochondrial DNA deletion(s) related myopathy rhabdomyolysis led to cessation of KD. Three individuals with POLG mutations died while being on KD, however, their survival was not different compared to individuals with POLG mutations without KD.
\end{abstract}

Conclusion: Data on efficacy and safety of KD for MD is too scarce for general recommendations. KD should be considered in individuals with MD and therapy refractory epilepsy, while KD is contraindicated in mitochondrial DNA deletion(s) related myopathy. When considering $\mathrm{KD}$ for $\mathrm{MD}$ the high rate of adverse effects should be taken into account, but also spectacular improvements in individual cases. KD is a highly individual management option in this fragile patient group and requires an experienced team. To increase knowledge on this—individually_promising management option more (prospective) studies using adequate outcome measures are crucial.

Keywords: Epilepsy, Complex I, Treatment, Management, Mitochondrial myopathy, OXPHOS, Mitochondrial DNA deletion, Adverse event, Modified Atkins diet, High fat diet

*Correspondence: Heidi.Zweers-vanEssen@radboudumc.nl

${ }^{\dagger}$ Heidi Zweers and Annemiek M. J. van Wegberg have contributed equally to this work

${ }^{1}$ Department of Gastroenterology and Hepatology - Dietetics,

Radboudumc, Postbus 9101, 6500 HB Nijmegen, The Netherlands

Full list of author information is available at the end of the article

\section{Introduction}

Mitochondrial diseases (MDs) are a heterogenous group of inborn metabolic diseases caused by defects in the genes encoding mitochondrial proteins that are required for ATP production from oxidation of substrates via the tricarboxylic acid cycle and the oxidative phosphorylation (OXPHOS). Underlying pathogenic variants can be found in nuclear or mitochondrial DNA (mtDNA) [1, 2]. Currently more than 295 different disorders are known 
[2]. Virtual all the extremely heterogeneous symptoms can occur with onset at all ages, but typically tissues with high energy requirements like skeletal/heart muscle and brain are mainly affected. Currently no curative treatment is available, making the supportive management for symptom relief priority.

Given the key role of mitochondria in energy metabolism and the importance of vitamins and co-factors for proper mitochondrial function, nutritional interventions are an integral component of daily management [3-7]. Moreover, nutritional interventions focusing on nutritional status or gastro-intestinal complaints have been shown effective [3, 4].

A ketogenic diet (KD) is a low-carbohydrate highfat diet that shifts metabolism towards $\beta$-oxidation and ketone body production. Three kind of KDs are defined. The classic KD uses grams of fat: grams of carbohydrate plus protein-ratio (e.g. 4:1 or 3:1) in every meal. Fat can be (mainly) given as medium-chain triglyceride (MCT), this subtype is called MCT-KD. As MCT-fats are converted easier into ketones than longer chain fatty acids, ketosis can be achieved more easily and more carbohydrates can be consumed. In contrast, the modified Atkins diet (MAD) only restricts carbohydrates (10-20 g per day) without restricting the amount of fat and protein [8-10].

KDs have been proven successful in the treatment of intractable epilepsy and are generally well tolerated and safe $[9,11,12]$. It is thought that KDs exert their positive effect (among others) via stimulation of mitochondrial biogenesis, improvement of mitochondrial function and decrease of oxidative stress [13-16] and therefore have been implemented in some cases with MD and epilepsy $[12,17]$. There are also studies suggesting a potential beneficial effect of KD in MD, besides reducing seizures [14, $18]$. However, this was mainly studied in patient derived fibroblasts and animal models [8, 14, 19-23]. Of note, while it was previously assumed that the liver provides ketone bodies to the brain, astrocytes itself have shown to be ketogenic cells. This astrocyte ketogenesis might control the survival/death decision of neural cells at least twofold. By scavenging non-esterified fatty acids the ketogenic pathway could prevent the detrimental actions of these metabolites and their derivatives (e.g. ceramide) on brain structure and function. Further, by acting directly as pro-survival metabolites, the ketone bodies may preserve neuronal synaptic function and structural stability [24].

When a diet provides only small amounts of glucose, hormones as glucagon inhibit glycolysis and stimulate ketogenesis. These ketone bodies can only be produced in the liver and in astrocytes, and provide the mitochondrial OXPHOS with a substrate for energy production.
The fatty acid pathway provides 5.7 times more flavin adenine dinucleotide (FADH2) than the glycose pathway and therefore fat has a potential benefit over carbohydrates as an energy substrate in human complex 1 deficiency [25]. However nicotinamide-adenine-dinucleotide (NADH) is still formed from all substrates and complex 1 is never completely bypassed (Fig. 1).

Pyruvate dehydrogenase complex (PDHC) deficiency hampers the conversion of pyruvate to acetyl-CoA and KDs are the pathomechanism based therapy as ketones, converted to acetyl-CoA, bypass the PDHC [26]. Pyruvate Carboxylase deficiency is a contraindication for $\mathrm{KD}$ as gluconeogenesis is impaired and affected individuals depend on nutritional glucose.

Taken together, KDs are an interesting management option for MD that needs further evaluation. We here perform a systematic literature review to assess efficacy and safety of KD for MD.

\section{Methods \\ Search strategy}

This systematic review was conducted according to the Preferred Reporting Items for Systematic Reviews and Meta-Analyses (PRISMA) guidelines [27]. We identified relevant studies using medical subject headings (MeSH) and text words related to KD and MD (see Additional file 1). The databases searched were: Pubmed, Cochrane, Embase and Cinahl (November 2020) without any search limits. The search strategy for Pubmed was generated together with a specialist librarian and accordingly amended for the other databases.

\section{Study selection}

Two authors (HZ, AvW) independently screened and selected the papers using Rayyan ${ }^{\circledR}$ [28]. Eligibility criteria were: cases with MD using a KD and English language. The same authors reviewed full-texts of these selected papers independently, according to exclusion criteria. Disagreements were resolved by consensus and discussion with a third author (SBW). Exclusion criteria were: (i) cases without genetically proven MD [1, 2], (ii) cases with PDHC-deficiency, (iii) cases not on KD or without details of the KD composition and (iv) cases without data of effect on clinical phenotype before and under treatment.

KD was defined as any dietary manipulation of fat, carbohydrate and protein aiming to achieve ketosis and included the 'classic' KD, MCT-KD or MAD [8-10]. High fat diets including the low glycaemic index diet are not likely to achieve ketosis and therefore cases treated with these diets were excluded.

Reference lists were reviewed for additional publications. 


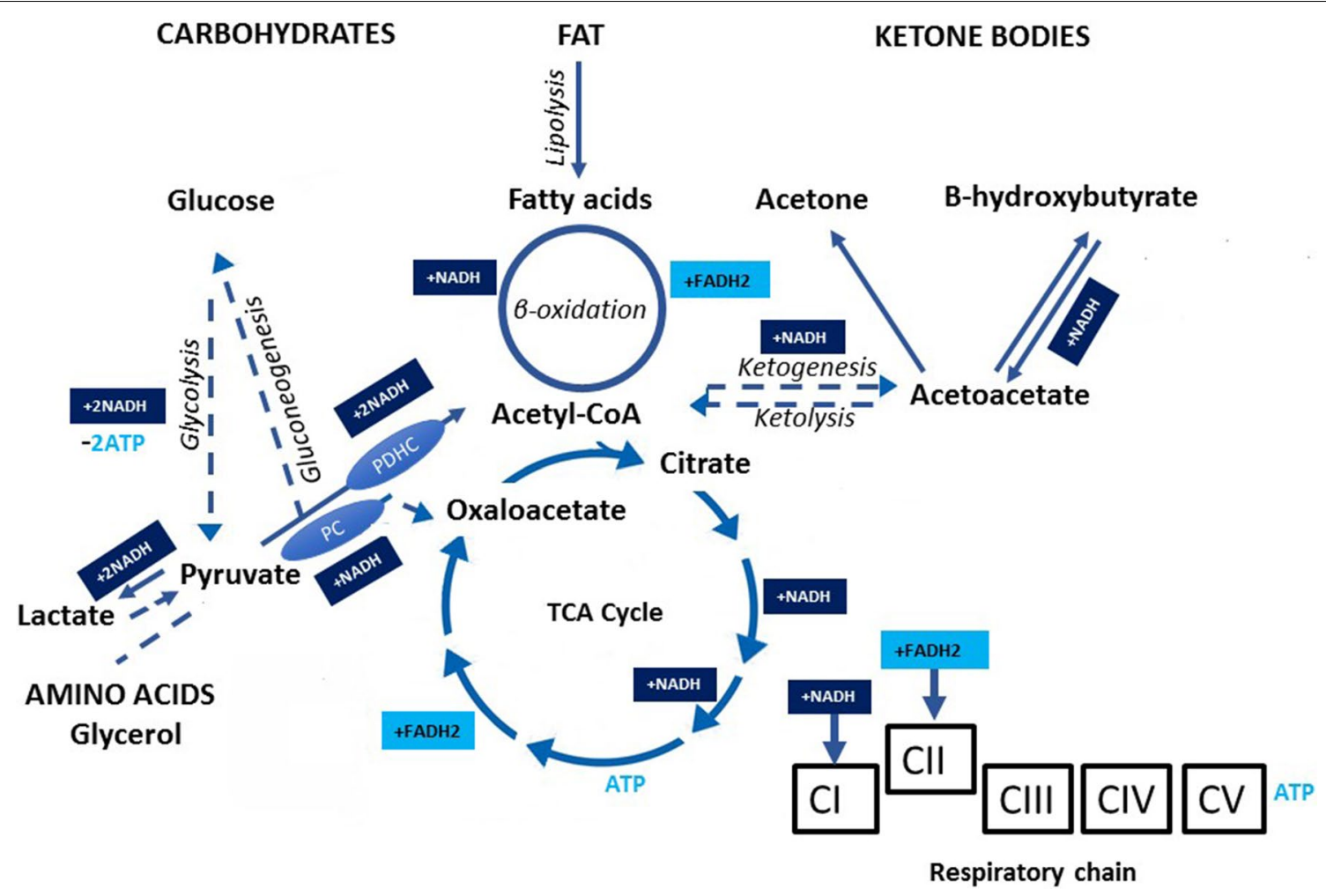

Fig. 1 Metabolic pathways of carbohydrates, fat and ketone bodies in energy metabolism. ATP adenosine triphosphate, C respiratory chain complex, PDHC pyruvate dehydrogenase complex, PC pyruvate carboxylase, TCA Cycle Tricarboxylic acid cycle also called citric acid cycle

\section{Outcome measures}

The primary outcome was the effect of KD on clinical phenotypes (epilepsy, muscle involvement, tonus dysregulation (muscular hyper- or hypotonia), movement disorders, developmental delay and intellectual disability (DD/ID), other individual signs and symptoms) and the occurrence of adverse events (AEs). The secondary outcome was defined as the effect of KD on MRI findings and laboratory values (e.g. lactic acidosis, liver function test).

\section{Data extraction and quality appraisal}

Two authors (HZ, AvW) extracted data and checked the data for completeness. A third author (SBW) checked all articles again to ensure correct interpretation of data. Discrepancies were resolved through discussion and consensus.

We used The Oxford Levels of Evidence 2 [29] scoring to assess study quality as well as the The Risk Of Bias In Non-randomized Studies-of Interventions (ROBINSI) assessment tool [30]. No studies were suitable for pooling of the results and therefore a narrative analysis is presented.

\section{Results}

The search strategy yielded 1149 abstracts (PRISMA flowchart, Fig. 2 [31]) of which 17 papers reporting 20 cases were included in the detailed analysis. All data are summarized in Table 1 and Fig. 3. Of note, (multi)vitamins and other food supplements were reported in many of the included cases (see Table 1 for details). With exception of one case (TPK1 [32]) clinically not responding to thiamine supplementations, none of the reported vitamins or co-factors were pathomechanism based treatment options and therefore were not taken into account in our analysis.

\section{Case characteristics}

Of the 20 cases (12 female), 16 had a nuclear DNA and 4 a mtDNA related mutation. Of note 3 of the nuclear variants (TWNK) lead to multiple mtDNA deletions. The ages at start of KD ranged between 0 and 62 years ( 1 neonate (aged 7 days), 2 infants (9 and 10 months), 11 children (1.3-16 years), 6 adults (aged 22-62 years)). Eight individuals (8/20), were described as having epilepsy. In 10/20 individuals muscle involvement (cardiomyopathy, muscle weakness, exercise intolerance, ptosis) was reported, 7/20 were 

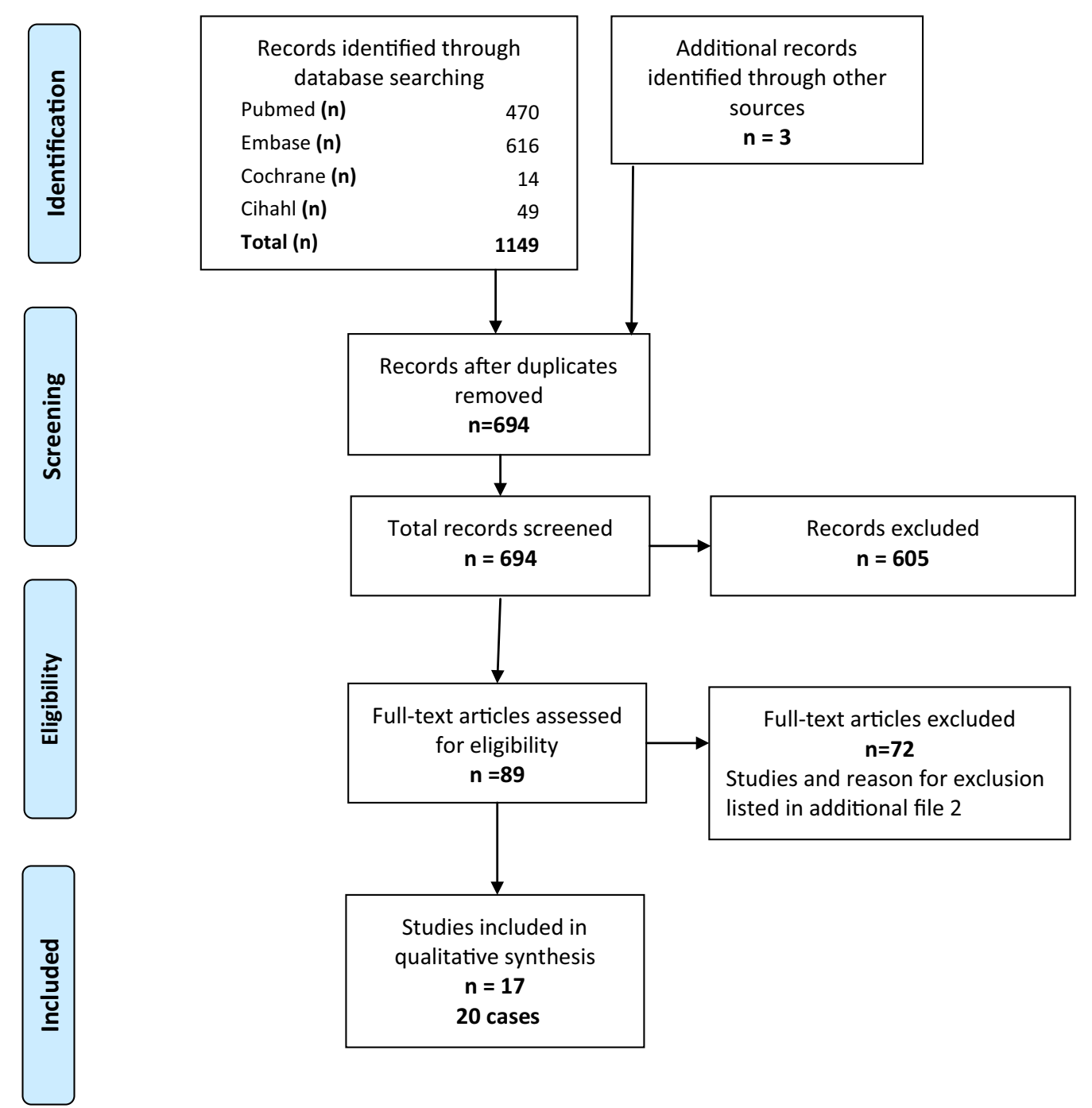

Fig. 2 PRISMA flowchart. This figure detailing the search strategy

described having tonus dysregulation, and for $5 / 20$ cases movement disorder(s) (ataxia, dystonia) were reported. In 10/20 cases DD/ID was reported. Other reported symptoms were visual problems, respiratory distress, headaches/migraine, failure to thrive, feeding problems, gastro-intestinal problems, alopecia and hearing loss.

\section{Study quality}

The general study quality was low, with 15 case reports (category 4) and one controlled trial with 5 adult participants (category $3 \mathrm{~b}$ ). The risk of bias in the trial was scored as low.

\section{Interventions}

Eleven cases followed a classical KD, 8 cases a MAD. For one case the composition of the KD was not detailed, however, this case was included as achievement of ketosis was documented. For a total of 14 cases achievement of ketosis was reported. The total diet duration of all 20 cases together was $>22$ patient years, the median diet duration was 4 months (range 4 days -9 years). The main reasons for discontinuation of the $\operatorname{diet}(\mathrm{n}=12)$ were death $(n=3)$ [33-35] and other AEs $(n=7)$ [36-39]. In one case the daily constraints [40] and in one case the child's craving for carbohydrates and lack of improvement of hearing [41] lead to cessation after 2 years and 4 months, respectively. 


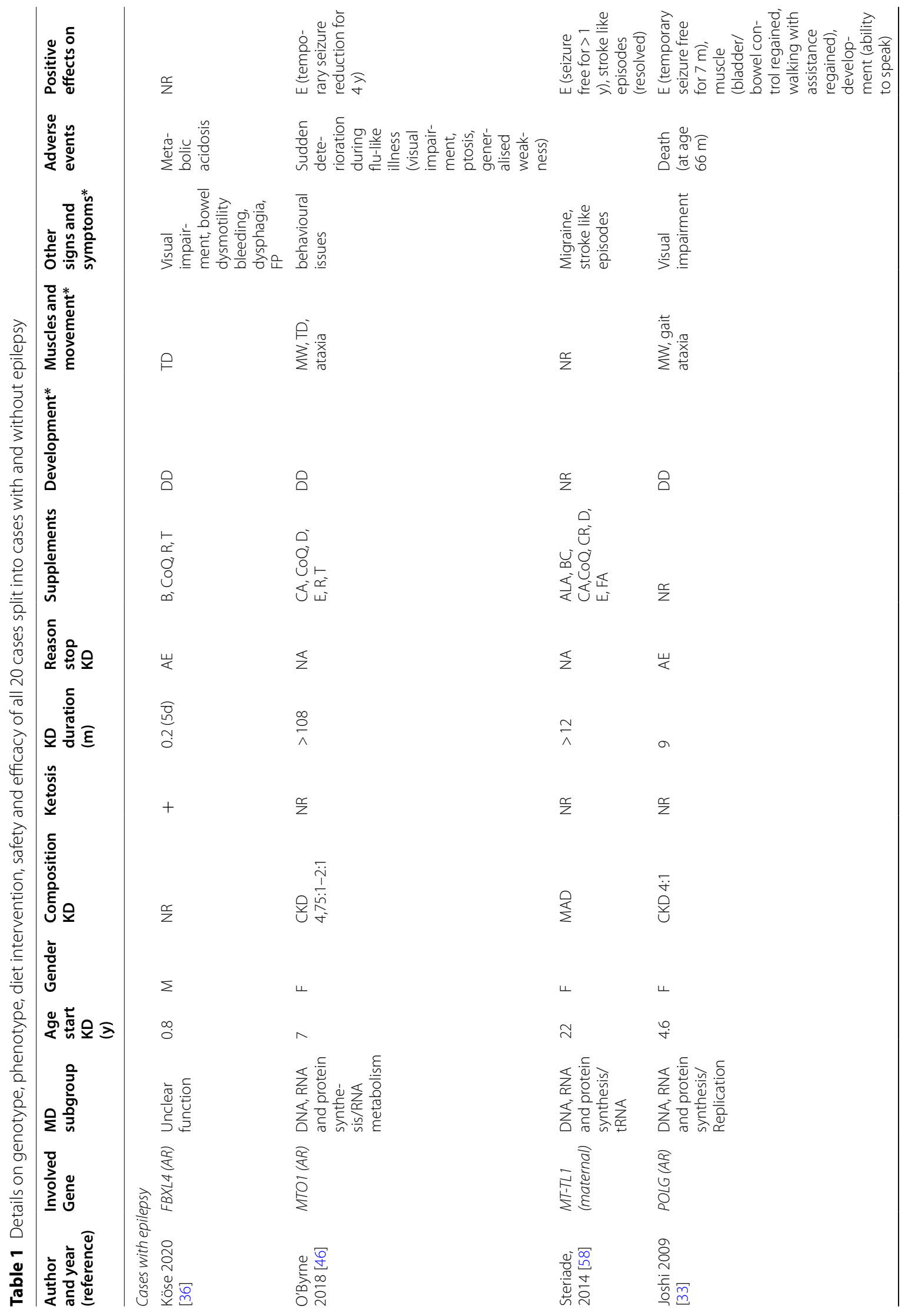




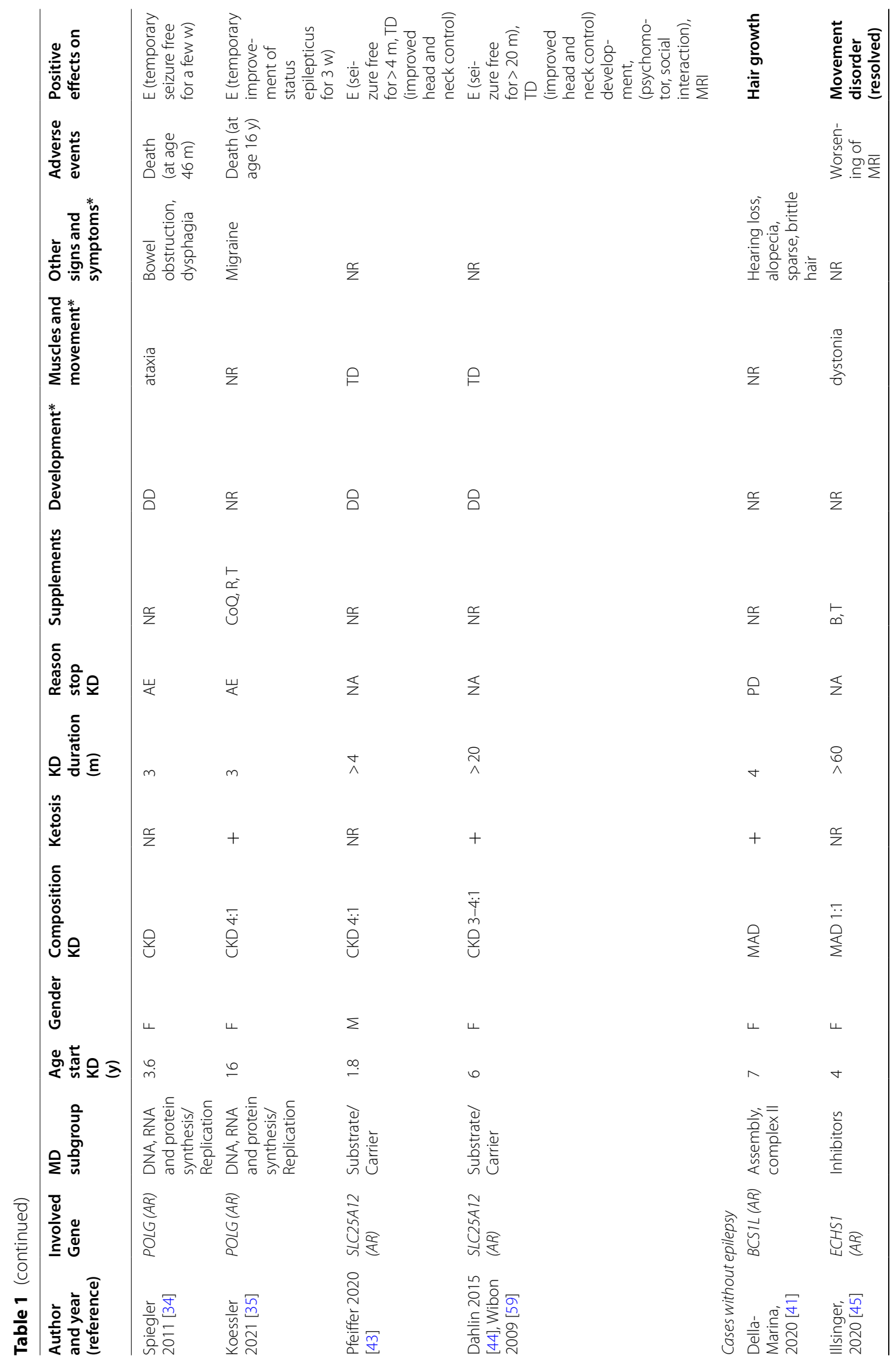




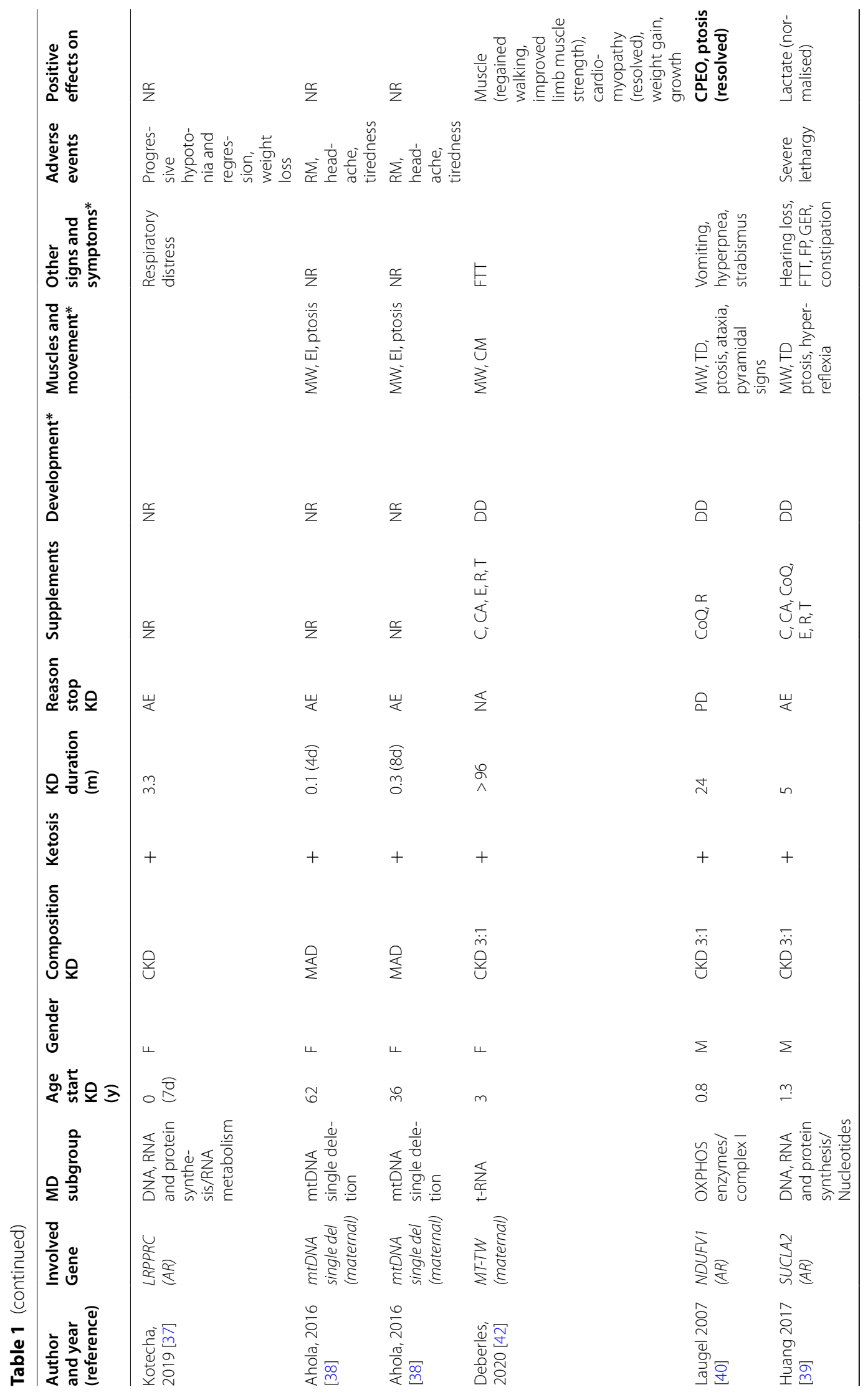




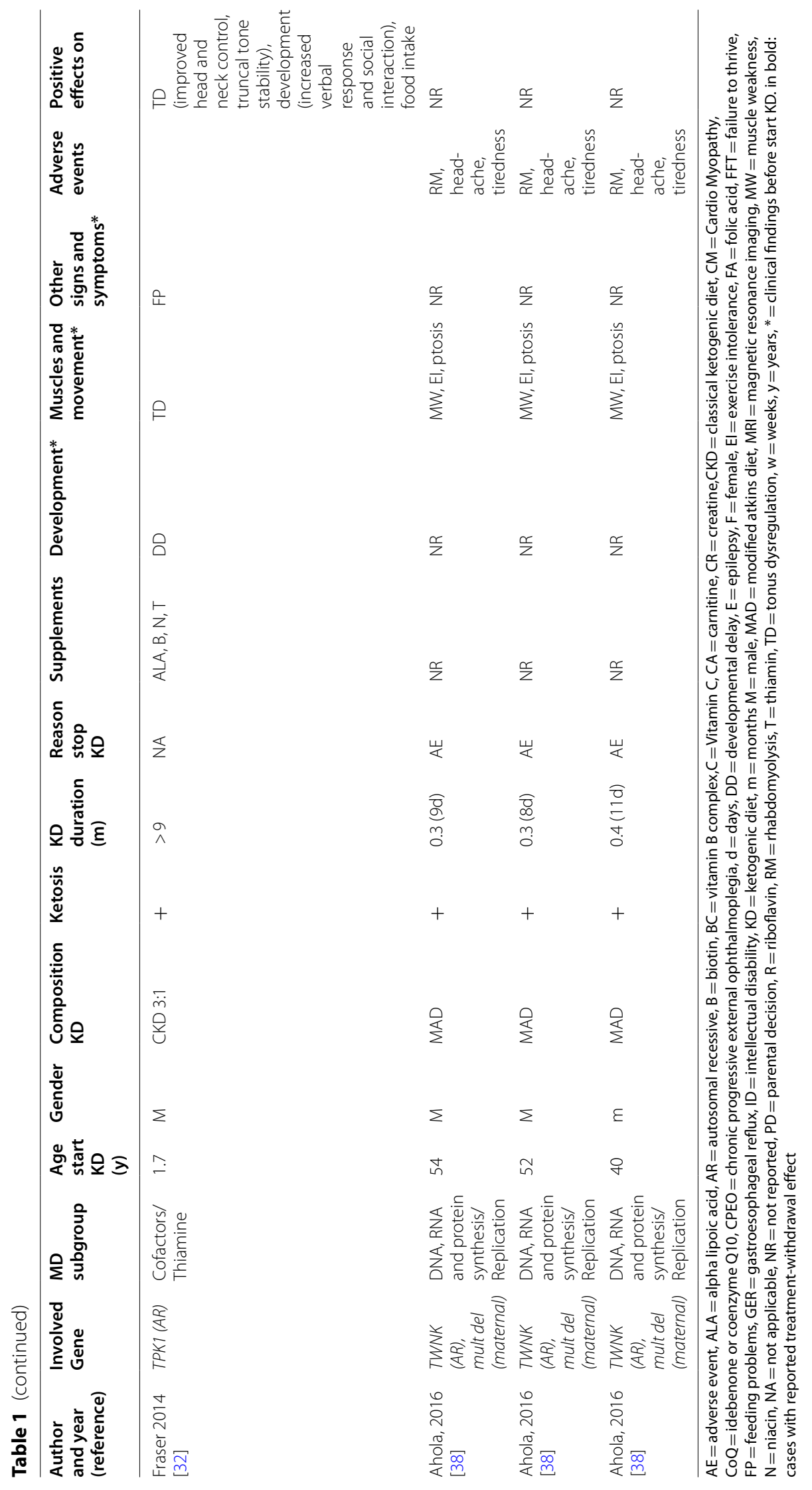




\section{Primary outcomes \\ Effect of $K D$ on epilepsy}

In $7 / 8$ cases with uncontrolled epilepsy seizure control was achieved with KD (5 seizure free, 1 reduction of seizures and 1 "stabilisation" of status epilepticus). In 4 of these cases the effect was only temporary (lasting a few weeks to 7 months). In one case no positive effect of the KD on epilepsy was reported, and the KD was stopped after 5 days due to metabolic acidosis.

\section{Effect of $K D$ on other clinical signs and symptoms}

Skeletal and heart muscle In 3/10 cases with muscle involvement KD had a positive effect. This was the return of bladder and bowel control, and the ability to walk with assistance [33], and the complete resolve of chronic progressive ophthalmoplegia (CPEO) and ptosis [40]. In the third case, the 3-year-old individual regained walking abilities with improved lower limb muscle strength after one year of KD and normalisation of septum thickness of hypertrophic cardiomyopathy after 3 years of KD. This effect was sustained at the last follow up at age 11 years (total 8 years of KD) [42]. A negative effect was observed in 6/10 individuals. Five of these six individuals experienced rhabdomyolysis, headache and fatigue leading to cessation of KD after 4 to 11 days [38] and in one case progressive muscular hypotonia with swallowing difficulties was reported going along with a weight loss below the $3^{\text {rd }}$ percentile [37].

Tonus dysregulation Tonus dysregulation was reported to improve in $3 / 7$ cases, while in one case progressive muscular hypotonia was only reported after the KD was started [37]. In all 3 cases head control improved [32, 43, 44 ] and one case additionally showed improved stability of truncal tone enabling him to stand with support and to sit independently [32].

Movement disorder In one case the paroxysmal ophisthotonic dystonia completely resolved for more than 5 years of follow-up [45]. In one individual the ataxia did not improve [46], in the other 3 cases no further details were provided $[33,34,40]$.

Developmental delay/Intellectual disability In $3 / 10$ cases with DD/ID a positive effect of KD was reported (e.g. increased verbal response and abilities, social interaction, improved memory) however no data of formal psychological or developmental testing were presented $[32,33,44]$. The other publications did not provide further details.
Other An improved oral food intake [32], improved weight gain and growth [42] and hair growth in an individual with alopecia [41] were reported in one case each.

Treatment-withdrawal effect In 3 cases a treatment withdrawal effect was demonstrated. In the case where KD had resulted in hair growth, this was lost again 6 months after cessation of KD [41]. In the individual where KD led to dissapearance of the dystonic-opisthotonic episodes within 5 days for 5 months, these reoccured within a few days when the KD was stopped, and dissapeared again 4 weeks after reintroducing KD [45]. In the case where CPEO and ptosis resolved within days and remained absent for 2 years on KD, the ptosis partially reoccured upon "relaxation" of the diet [40].

\section{Adverse events}

In 13/20 individuals AEs were reported (Table 1). In one study [38] all 5 adult participants with mitochondrial myopathy (2 mtDNA single deletion, $3 \mathrm{TWNK} / \mathrm{multi}$ ple mtDNA deletions) stopped MAD within 4-11 days because of rhabdomyolysis, headache and tiredness.

Three individuals, all with POLG-related Alpers syndrome, died during KD (at the ages of 46 months [34], 66 months [33] and 16 years [35]) of respiratory failure $(n=2)$ or paralytic bowel obstruction $(n=1)$. This was 3 months $(n=2)[34,35]$ and 35 months [33] respectively, after presentation.

Other AEs that lead to immediate cessation of the KD were severe lethargy, which occurred after 5 months of $\mathrm{KD}$ in one case [39] and lactic acidosis (after 5 days of KD) in one other case [36]. Of note, two individuals in two reports remained on KD despite AEs. In one case because of successful seizure reduction despite a sudden deterioration of visual acuity, ptosis and general weakness after 6 months of KD [46] and in one case the resolve of the movement disorder outweighed the worsening seen on MRI [45]. In one individual [37] who suffered from weight loss, regression and hypotonia the KD was continued for 3.3 months and then weaned to a more conventional feeding regime.

\section{Secondary outcomes}

From the 12 cases with reported MRI abnormalities for only 2 cases MRI details were provided after KD initiation. In one case a resumed myelination during KD was observed [44], while in the second the MRI worsened [45]. Interestingly in the latter case, the movement disorder had completely resolved and the patient was reported to develop age-adequately. In 7 cases lactic acidosis was reported which normalised in one [39], worsened in one [36] and did not change in 2 cases [37, 40]. For the remaining 3 cases no details were reported $[42,43,46]$. 


\begin{tabular}{|c|c|c|c|c|}
\hline \multicolumn{2}{|c|}{ Negative effect of KD (AE): } & Gene (reference) & Positive effect of & KD on: \\
\hline \multicolumn{5}{|c|}{ CASES WITH EPILEPSY } \\
\hline & metabolic acidosis & $F B X L 4(36)$ & & \\
\hline & deterioration & MTO1(46) & epilepsy* & \\
\hline & & MT-TL1 (58) & epilepsy seizure free & SLE resolved \\
\hline & death & POLG(33) & epilepsy seizure free* & development SM: B/B control, walking \\
\hline & death & POLG(34) & epilepsy seizure free * & \\
\hline & death & POLG(35) & epilepsy* & \\
\hline & & SLC25A12 (43) & epilepsy seizure free & TD: HC \\
\hline & & $S L C 25 A 12(44)$ & epilepsy seizure free & development TD: HC MRI \\
\hline \multicolumn{5}{|c|}{ CASES WITHOUT EPILEPSY } \\
\hline & & $B \operatorname{CS} 1 L(41)$ & hair growth & \\
\hline & MRI & ECHS1 (45) & movement disorder & \\
\hline \multirow{7}{*}{$\begin{array}{l}\text { hypotoniaregression } \\
\text { headache fatigue } \\
\text { headache fatigue }\end{array}$} & weight loss & $\operatorname{LRPPRC}(37)$ & & \\
\hline & SM: rhabdomyolysis & $m t D N A$ single del (38) & & \\
\hline & SM: rhabdomyolysis & $m t D N A$ single del (38) & & \\
\hline & & $M T-T W(42)$ & SM: walking & heart muscle weight/growth \\
\hline & & NDUFV1(40) & SM:CPEO/ptosis & \\
\hline & lethargy & SUCLA2 (39) & lactate & \\
\hline & & TPK1 (32) & TD:HC and truncal tone & development food intake \\
\hline headache fatigue & SM: rhabdomyolysis & TWNK, mult mtDNA del (38) & & \\
\hline headache fatigue & SM: rhabdomyolysis & TWNK, mult mtDNA del (38) & & \\
\hline headache fatigue & SM: rhabdomyolysis & TWNK, mult mtDNA del (38) & & \\
\hline \multicolumn{5}{|c|}{$\begin{array}{l}\text { Fig. } 3 \text { Summary of positive and negative effects of ketogenic diet in } 20 \text { cases with genetically proven mitochondrial disease. This figure visualises } \\
\text { the negative effects and adverse events on the left and the positive effects (on the right) of ketogenic diet in } 20 \text { cases with genetically proven } \\
\text { mitochondrial disease. *temporary effect }{ }^{* *} \text { cases with reported treatment-withdrawal effect. B/B bladder and bowel, CPEO chronic progressive } \\
\text { external ophthalmoplegia, del deletions, HC head control, mult multiple deletions in mitochondrial DNA, MRI magnetic resonance imaging, mtDNA } \\
\text { mitochondrial DNA, SLE stroke like episodes, SM skeletal muscle, TD tonus dysregulation }\end{array}$} \\
\hline
\end{tabular}

Two individuals with POLG mutations had mildly elevated liver function tests before KD initiation [33, 34]. In one case these remained elevated [33], in the other case no details were provided [34]. In one individual a transient, four day long, increase in liver function tests occurred [35].

\section{Discussion}

Despite identifying 694 studies using our search strategy, only 20 cases (one controlled trial $(n=5)$ and 15 case reports) were of sufficient quality for detailed analysis. These data are too scarce to draw firm conclusions regarding efficacy and safety of KD. Future reports on KD for MDs must present a minimum of "common data elements" (in line with the data shown in Table 1) describing the composition of KD as well as the clinical effect including adverse events.

\section{KD is effective for seizure control in MD}

KD was highly effective and led to seizure control of therapy refractory seizures in 7 of $8 \mathrm{MD}$ cases, at least temporarily. KD was stopped only after 5 days in the 8 th case and it remains elusive if adaptation of the KD would have overcome the occurring lactic acidosis and would have led to seizure control. The number of cases does not allow comparing with the efficacy of KD for intractable seizures of other causes (up to $55 \% / 25 \%$ becoming seizure free on 4:1 classical KD/MAD [9]).

KD might be effective for the treatment of other signs and symptoms of MD in individual cases, but is contraindicated in mtDNA deletion(s) related myopathy

In $12 / 20$ cases KD was initiated for other indications than epilepsy. In 5 adults with mitochondrial myopathy KD 
was stopped due to AEs in all participants [38]. However, a potential long term benefit cannot be excluded, as the authors report a slight improvement on muscle strength and in 6-min walking test in three of four patients after 2.5 years of follow up after cessation of KD.

In the remaining 7 pediatric cases, 5 improved clinically [32, 40-42, 45], mainly concerning muscle symptoms. Especially the well reported treatment-withdrawal effect in three cases (start/stop hair growth, resolving/ reoccurring ptosis or movement disorder) illustrates the potential for $\mathrm{KD}$ in individual management of $\mathrm{MD}$. Of note, in one case hypertrophic cardiomyopathy was completely resolved on KD and sustained without any additional medication [42]. The authors discuss that ketone bodies may have modulated cardiac metabolism. This is in line with the data suggesting that in heart failure due to metabolic dysfunction fatty acids allow for sufficient energy production while carbohydrates may contribute to declining contractile function. A role for ketones both in signalling as well as an energy source is suspected to underlie this [47].

\section{Safety aspects of KD for MD}

AEs occurred $65 \%$ of MD cases during KD. This percentage is comparable with studies on KD for PDHC deficiency $(13 / 19=68 \%)[26]$ or epilepsy with mitochondrial dysfunction $(22 / 34=65 \%)[12,17]$. AEs of KD reported in literature for refractory epilepsy are mainly gastrointestinal complaints rarely leading to discontinuing of the diet, but also lethargy and acidosis have been reported [9, 11 .

The 3 children with MD that died (aged 66-192 months) while being on KD all had POLGrelated Alpers disease, an early lethal disorder with a median age at death of 16 (range 1-181) months [48]. Hence, their age of death is comparable. Moreover, the median survival after presentation is reported to be 5 $(0.5-181)$ months without KD [48], and was $3(n=2)$ and 35 months, in the cases with KD reviewed here.

Hence, from these limited data it seems unlikely that KD negatively influenced mortality, but is in line with the natural disease course of childhood onset MD.

\section{Practical recommendations}

The current guidelines on KD list complex I deficiency as a condition for which KD has been shown reportedly more beneficial when compared to the average response to KD in refractory epilepsy [11, 12, 49] in general. This pathomechanism approach assumes that fatty acids compared to carbohydrates produce more FADH that can enter complex II $[12,18,49,50]$ and hence allows (partial) bypassing of complex I. However, as outlined in the introduction (Fig. 1), NADH is still formed from all substrates and complex I is never completely bypassed.

Our study did solely include cases with known genetic background as increasing knowledge from next generation studies shows that complex I deficiency cannot only be seen in MD but also in other genetic diseases especially if measured in muscle specimen of patients with terminal disease [51]. Hence, there is insufficient evidence that $\mathrm{KD}$ is more beneficial in mitochondrial complex I related disease than in other MD [11] or even other therapy refractory epilepsies. However, from our results we conclude that KD should be considered in MD patients with therapy resistant epilepsy.

Given the risk of AEs KD should be initiated by a team experienced with both MD and KD. Especially in the first weeks clinical and laboratory controls should be frequent (in line with the general guideline on KD). From our data we cannot conclude after which duration the efficacy can be judged and we therefore recommend a three months trial of KD, in analogy to KD for intractable epilepsy [52]. Whether classic KD or MAD is superior is unknown and an individual top-down (start KD 4:1) or bottom up approach (start with MAD) should be weighed and discussed with patient and/or parents.

\section{An appraisal for high fat diets}

In this context, we would like to mention the high fat diets. The beneficial effects of KD for MDs are probably not only based on ketogenesis and energy expenditure from ketone bodies $[22,53]$. Two studies that did not met our inclusion criteria, reporting $4 \mathrm{MD}$ cases with complex I deficiency in muscle without a genetic diagnosis, showed improved maximal workload and muscle force under high fat diet $[54,55]$. Another $n=1$ trial reported a high fat diet improving the endurance in a bicycle test when compared to a high carbohydrate diet in one adult (TMEM126B) [50].

There is further interesting evidence to encourage human studies on high fat diets for MD First, supplementing of complex I deficient human fibroblast cell lines with palmitate resulted in protection from cell death caused by glucose withdrawal presumably based on fatty acid induced stimulation of mitochondrial biogenesis. Second, the study of a mouse model of Harlequin complex I deficient mice established that a high fat diet slowed down disease progression regarding major neurodegenerative symptoms and cerebellar atrophy [56].

More studies on high fat diet in humans reporting the aforementioned common data elements are necessary to draw conclusions. However, it also has to be considered that a high-fat diet could downregulate genes involved in 
the mitochondrial respiratory chain, and could thereby worsen the mitochondrial dysfunction [57].

\section{Conclusion}

Data on efficacy and safety of KD for MD is too scarce for general recommendations. KD should be considered in individuals with MD and therapy refractory epilepsy, while mtDNA deletion(s) related myopathy is a contraindication (as well as Pyruvate Carboxylase deficiency). KD is a highly individual management option in this fragile patient group and requires an experienced team. To increase knowledge on thisindividually-promising management option more (prospective) high quality studies using adequate outcome measures are crucial.

\section{Abbreviations}

AE: Adverse event; KD: Ketogenic diet; MeSH: Medical subject headings; MD: Mitochondrial disease; MAD: Modified Atkins diet; OXPHOS: Oxidative phosphorylation; PRISMA: Preferred Reporting Items for Systematic Reviews and Meta-Analyses; PDHC: Pyruvate Dehydrogenase Complex.

\section{Supplementary Information}

The online version contains supplementary material available at https://doi. org/10.1186/s13023-021-01927-w.

Additional file 1. Search strategy.

Additional file 2. Excluded studies.

\section{Acknowledgements}

We thank Leo Kluijtmans, PhD, Clinical Laboratory Geneticist, Radboudumc, Nijmegen, the Netherland for critical review of Fig. 1. We thank Elles v.d. Louw, PhD, dietician, Erasmusmc, Rotterdam, The Netherlands for fruitful discussion about common data elements in reporting studies on ketogenic diet. We thank Elmie Peters, librarian, Radboudumc, Nijmegen, The Netherland for her assistance with the search strategy.

\section{Authors' contributions}

All authors substantially contributed to the design of the study. HZ, AvW, SBW acquired, analysed and interpreted data. $\mathrm{HZ}$ and AvW drafted the work, all authors revised it critically for important intellectual content and approved the final version to be published. All authors agree to be accountable for all aspects of the work in ensuring that questions related to the accuracy or integrity of any part of the work are appropriately investigated and resolved. All authors read and approved the final manuscript.

\section{Funding}

SBW has the ERAPERMED2019-310 grant—Personalized Mitochondrial Medicine: Optimizing diagnostics and treatment for patients with mitochondrial diseases.

\section{Availability of data and materials}

All data generated or analysed during this study are included in this published article and its additional information files.

\section{Declarations}

Ethics approval and consent to participate Not applicable.
Consent for publication

Not applicable.

\section{Competing interests}

The authors declare that they have no competing interests.

\section{Author details}

'Department of Gastroenterology and Hepatology - Dietetics, Radboudumc, Postbus 9101, 6500 HB Nijmegen, The Netherlands. ${ }^{2}$ Radboud Center for Mitochondrial Medicine (RCMM), Amalia Children's Hospital, Radboudumc, Nijmegen, The Netherlands. ${ }^{3}$ University Children's Hospital, Paracelsus Medical University, Salzburg, Austria. ${ }^{4}$ Department of Internal Medicine, Radboudumc, Nijmegen, The Netherlands.

Received: 25 March 2021 Accepted: 13 June 2021

Published online: 03 July 2021

\section{References}

1. Thompson K, Collier JJ, Glasgow RIC, Robertson FM, Pyle A, Blakely EL, et al. Recent advances in understanding the molecular genetic basis of mitochondrial disease. J Inherit Metab Dis. 2020;43(1):36-50.

2. Wortmann SB, Mayr JA, Nuoffer JM, Prokisch H, Sperl W. A guideline for the diagnosis of pediatric mitochondrial disease: the value of muscle and skin biopsies in the genetics era. Neuropediatrics. 2017;48(04):309-14.

3. Zweers H, Smit D, Leij S, Wanten G, Janssen MCH. Individual dietary intervention in adult patients with mitochondrial disease due to the m.3243A>G mutation: the DINAMITE study, a randomized controlled trial. Nutrition. 2020;69:110544.

4. Schiff M, Bénit P, Coulibaly A, Loublier S, El-Khoury R, Rustin P. Mitochondrial response to controlled nutrition in health and disease2011 2011-1-1. $65-75 \mathrm{p}$

5. Wortmann SB, Essen HZ, Rodenburg RJT, Heuvel LPVANDEN, Vries MCDE, Rasmussen-conrad E, et al. Mitochondrial energy production correlates with the age-related BMI. Pediatric Res. 2009;65:103-8.

6. Repp BM, Mastantuono E, Alston CL, Schiff M, Haack TB, Rotig A, et al. Clinical, biochemical and genetic spectrum of 70 patients with ACAD9 deficiency: is riboflavin supplementation effective? Orphanet J Rare Dis. 2018;13(1):120.

7. Distelmaier F, Haack TB, Wortmann SB, Mayr JA, Prokisch H. Treatable mitochondrial diseases: cofactor metabolism and beyond. Brain J Neurol. 2017;140(2):e11.

8. Kossoff EH, Hartman AL. Ketogenic diets: new advances for metabolismbased therapies. Curr Opin Neurol. 2012;25(2):173-8.

9. Martin-McGill KJ, Bresnahan R, Levy RG, Cooper PN. Ketogenic diets for drug-resistant epilepsy. The Cochrane database of systematic reviews. 2020;6:CD001903.

10. Masino SA. Ketogenic Diet and Metabolic Therapies, Chapter 2. In: Masino SA, editor.: Oxford University Press Inc; 2016.

11. Kossoff EH, Zupec-Kania BA, Auvin S, Ballaban-Gil KR, Christina Bergqvist AG, Blackford R, et al. Optimal clinical management of children receiving dietary therapies for epilepsy: Updated recommendations of the International Ketogenic Diet Study Group. Epilepsia Open. 2018;3(2):175-92.

12. Kang H-C, Lee Y-M, Kim HD, Lee JS, Slama A. Safe and effective use of the ketogenic diet in children with epilepsy and mitochondrial respiratory chain complex defects. Epilepsia. 2007:48(1):82-8.

13. Stafford P, Abdelwahab MG, Kim DY, Preul MC, Rho JM, Scheck AC. The ketogenic diet reverses gene expression patterns and reduces reactive oxygen species levels when used as an adjuvant therapy for glioma. Nutr Metab (Lond). 2010;7:74.

14. Ahola-Erkkila S, Carroll CJ, Peltola-Mjosund K, Tulkki V, Mattila I, SeppanenLaakso T, et al. Ketogenic diet slows down mitochondrial myopathy progression in mice. Hum Mol Genet. 2010;19(10):1974-84.

15. Bough KJ, Wetherington J, Hassel B, Pare JF, Gawryluk JW, Greene JG, et al. Mitochondrial biogenesis in the anticonvulsant mechanism of the ketogenic diet. Ann Neurol. 2006;60(2):223-35.

16. Danial NN, Hartman AL, Stafstrom CE, Thio LL. How does the ketogenic diet work? Four potential mechanisms. J Child Neurol. 2013;28(8):1027-33. 
17. Na J, Kim H, Lee Y. Effective and safe diet therapies for Lennox-Gastaut syndrome with mitochondrial dysfunction. Therapeutic Advances in Neurological Disorders. 2020;13.

18. Roef MJ, de Meer K, Reijngoud D-J, Straver HWHC, de Barse M, Kalhan SC, et al. Triacylglycerol infusion improves exercise endurance in patients with mitochondrial myopathy due to complex I deficiency. Am J Clin Nutr. 2002;75:237-44.

19. Emperador S, Lopez-Gallardo E, Hernandez-Ainsa C, Habbane M, Montoya J, Bayona-Bafaluy MP, et al. Ketogenic treatment reduces the percentage of a LHON heteroplasmic mutation and increases mtDNA amount of a LHON homoplasmic mutation. Orphanet J Rare Dis. 2019:14(1):150.

20. Sullivan PG, Rippy NA, Dorenbos K, Concepcion RC, Agarwal AK, Rho $J M$. The ketogenic diet increases mitochondrial uncoupling protein levels and activity. Ann Neurol. 2004;55(4):576-80.

21. Hughes SD, Kanabus M, Anderson G, Hargreaves IP. The ketogenic diet component decanoic acid increases mitochondrial citrate synthase and complex I activity in neuronal cells. J Neurochem. 2014;129(3):426-33.

22. Hasan-Olive MM, Lauritzen KH, Ali M, Rasmussen LJ, Storm-Mathisen J. A ketogenic diet improves mitochondrial biogenesis and bioenergetics via the PGC1 alpha-SIRT3-UCP2 axis. Neurochem Res. 2019;44(1):22-37.

23. Maalouf M, Sullivan PG, Davis L, Kim DY, Rho JM. Ketones inhibit mitochondrial production of reactive oxygen species production following glutamate excitotoxicity by increasing NADH oxidation. Neuroscience. 2007;145(1):256-64.

24. Guzman M, Blazquez C. Ketone body synthesis in the brain: possible neuroprotective effects. Prostaglandins Leukot Essent Fatty Acids. 2004;70(3):287-92.

25. Rahman S. Mitochondrial disease and epilepsy. Dev Med Child Neurol. 2012;54(5):397-406.

26. Sofou K, Dahlin M, Hallbook T, Lindefeldt M, Viggedal G, Darin N. Ketogenic diet in pyruvate dehydrogenase complex deficiency: shortand long-term outcomes. J Inherit Metab Dis. 2017;40(2):237-45.

27. Liberati A, Altman DG, Tetzlaff J, Mulrow C, Gotzsche PC, loannidis JP, et al. The PRISMA statement for reporting systematic reviews and meta-analyses of studies that evaluate healthcare interventions: explanation and elaboration. BMJ. 2009;339:2700.

28. Ouzzani M, Hammady H, Fedorowicz Z, Elmagarmid A. Rayyan-a web and mobile app for systematic reviews. Syst Rev. 2016;5(1):210.

29. OCEBM Levels of Evidence Working Group* OCEBM Levels of Evidence Working Group = Jeremy Howick ICJLL, Paul Glasziou, Trish Greenhalgh, Carl Heneghan, Alessandro Liberati, Ivan Moschetti, Bob Phillips, Hazel Thornton, Olive Goddard and Mary Hodgkinson The Oxford Levels of Evidence 22011 [Available from: https://www.cebm.ox.ac.uk/ resources/levels-of-evidence/ocebm-levels-of-evidence

30. Sterne JAC HM, Reeves BC, Savović J, Berkman ND, Viswanathan M, Henry D, Altman DG, Ansari MT, Boutron I, Carpenter JR, Chan AW Churchill R, Deeks JJ, Hróbjartsson A, Kirkham J, Jüni P, Loke YK, Pigott TD, Ramsay CR, Regidor D, Rothstein HR, Sandhu L, Santaguida PL, Schünemann HJ, Shea B, Shrier I, Tugwell P, Turner L, Valentine JC, Waddington $\mathrm{H}$, Waters E, Wells GA, Whiting PF, Higgins JPT. ROBINS-I: a tool for assessing risk of bias in non-randomized studies of interventions. BMJ (Clinical research ed). 2016;355:14919

31. Moher D, Liberati A, Tetzlaff J, Altman DG, Group P. Reprint-preferred reporting items for systematic reviews and meta-analyses: the PRISMA statement. Phys Ther. 2009;89(9):873-80.

32. Fraser JL, Vanderver A, Yang S, Chang T, Cramp L, Vezina G, et al. Thiamine pyrophosphokinase deficiency causes a Leigh Disease like phenotype in a sibling pair: identification through whole exome sequencing and management strategies. Mol Genet Metab Rep. 2014;1:66-70.

33. Joshi CN, Greenberg CR, Mhanni AA, Salman MS. Ketogenic diet in Alpers-Huttenlocher syndrome. Pediatr Neurol. 2009;40(4):314-6.

34. Spiegler J, Stefanova I, Hellenbroich Y, Sperner J. Bowel obstruction in patients with Alpers-Huttenlocher syndrome. Neuropediatrics. 2011;42(5):194-6.
35. Koessler M, Haberlandt E, Karall D, Baumann M, Holler A, Scholl-Burgi $\mathrm{S}$. Ketogenic diet in a patient with refractory status epilepticus due to POLG mutation. JIMD Rep. 2021;57(1):3-8.

36. Kose E, Kose M, Edizer S, Akışın Z, Yilmaz ZB, Şahin A, et al. Different clinical presentation in a patient with two novel pathogenic variants of the FBXL4 gene. Turk J Pediatr. 2020;62(4):652-6.

37. Kotecha S, Kairamkonda V. Mitochondrial respiratory chain complex IV deficiency presenting as neonatal respiratory distress syndrome. BMJ Case Rep. 2019;12(7)

38. Ahola S, Auranen M, Isohanni P, Niemisalo S, Urho N, Buzkova J, et al. Modified Atkins diet induces subacute selective ragged-redfiber lysis in mitochondrial myopathy patients. EMBO Mol Med. 2016;8(11):1234-47.

39. Huang X, Bedoyan JK, Demirbas D, Harris DJ, Miron A, Edelheit S, et al. Succinyl-COA synthetase (SUCLA2) deficiency in two siblings with impaired activity of other mitochondrial oxidative enzymes in skeletal muscle without mitochondrial DNA depletion. 2017 2017-03-. Report No.: 1096-7206 Contract No.: 3.

40. Laugel V, This-Bernd V, Cormier-Daire V, Speeg-Schatz C, de SaintMartin A, Fischbach M. Early-onset ophthalmoplegia in Leigh-like syndrome due to NDUFV1 mutations. Pediatr Neurol. 2007;36(1):54-7.

41. Della Marina A, Leiendecker B, Roesch S, Wortmann SB. Ketogenic diet for treating alopecia in BCS1I-related mitochondrial disease (Bjornstad syndrome). JIMD reports. 2020;53(1):10-1.

42. Deberles E, Maragnes P, Penniello-Valette M-J, Allouche S, Joubert MA, Deberles E. Reversal of cardiac hypertrophy with a ketogenic diet in a child with mitochondrial disease and hypertrophic cardiomyopathy. Can J Cardiol. 2020;36(10):1690.

43. Pfeiffer B, Sen K, Kaur S, Pappas K. Expanding phenotypic spectrum of cerebral aspartate-glutamate carrier isoform 1 (AGC1) deficiency. Neuropediatrics. 2020:51(2):160-3.

44. Dahlin M, Martin DA, Hedlund Z, Jonsson M, von Dobeln U, Wedell A. The ketogenic diet compensates for AGC1 deficiency and improves myelination. Epilepsia. 2015;56(11):e176-81.

45. Illsinger S, Korenke GC, Boesch S, Nocker M, Karall D, Nuoffer JM, et al. Paroxysmal and non-paroxysmal dystonia in 3 patients with biallelic ECHS1 variants: Expanding the neurological spectrum and therapeutic approaches. Eur J Med Genet. 2020;63(11):104046.

46. O'Byrne JJ, Tarailo-Graovac M, Ghani A, Champion M, Deshpande C, Dursun A, et al. The genotypic and phenotypic spectrum of MTO1 deficiency. Mol Genet Metab. 2018;123(1):28-42.

47. Takahara S, Soni S, Maayah ZH, Ferdaoussi M, Dyck JRB. Ketone Therapy for Heart Failure: Current Evidence for Clinical Use. Cardiovasc Res. 2021.

48. Hikmat O, Tzoulis C, Klingenberg C, Rasmussen M, Tallaksen CME,

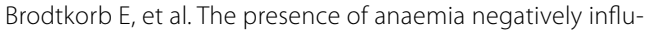
ences survival in patients with POLG disease. J Inherit Metab Dis. 2017;40(6):861-6.

49. Seo JH, Lee YM, Lee JS, Kim SH, Kim HD. A case of Ohtahara syndrome with mitochondrial respiratory chain complex I deficiency. Brain Dev. 2010;32(3):253-7.

50. Theunissen TEJ, Gerards M, Hellebrekers DMEl, van Tienen FH, Kamps $\mathrm{R}$, Sallevelt SCEH, et al. Selection and characterization of palmitic acid responsive patients with an OXPHOS complex I defect. Front Mol Neurosci. 2017;10:336.

51. Wortmann SB, Koolen DA, Smeitink JA, van den Heuvel L, Rodenburg RJ. Whole exome sequencing of suspected mitochondrial patients in clinical practice. J Inherit Metab Dis. 2015;38(3):437-43.

52. Vehmeijer FO, van der Louw EJ, Arts WF, Catsman-Berrevoets CE, Neuteboom RF. Can we predict efficacy of the ketogenic diet in children with refractory epilepsy? Eur J Paediatr Neurol. 2015;19(6):701-5.

53. Rho JM. How does the ketogenic diet induce anti-seizure effects? Neurosci Lett. 2017;637:4-10

54. de Meer K, Roef MJ, de Klerk JBC, Bakker HD, Smit GPA, Poll-The BT. Increasing fat in the diet does not improve muscle performance in patients with mitochondrial myopathy due to complex I deficiency. J Inherit Metab Dis. 2005;28:95-8. 
55. Roef MJ, de Meer K, Reijngoud D-J, Straver HWHC, de Barse M, Kalhan SC, et al. Triacylglycerol infusion does not improve hyperlactemia in resting patients with mitochondrial myopathy due to complex I deficiency. Am J Clin Nutr. 2002;75:228-36.

56. Schiff M, Benit P, El-Khoury R, Schlemmer D, Benoist JF, Rustin P. Mouse studies to shape clinical trials for mitochondrial diseases: high fat diet in Harlequin mice. PLoS ONE. 2011;6(12):e28823.

57. Sparks LM, Xie H, Koza RA, Mynatt R, Hulver MW, Bray GA, et al. A high-fat diet coordinately downregulates genes required for mitochondrial oxidative phosphorylation in skeletal muscle. Diabetes. 2005;54(7):1926-33.

58. Steriade C, Andrade DM, Faghfoury H, Tarnopolsky MA, Tai P. Mitochondrial encephalopathy with lactic acidosis and stroke-like episodes
(MELAS) may respond to adjunctive ketogenic diet. Pediatr Neurol. 2014;50(5):498-502.

59. Wibon R, Lasorsa F, Töhönen V, Barbaro M, Sterky F, Kucinski T et al. AGC1 deficiency associated with global cerebral hypomyelination. New Engl J Med. 2009:489-95.

\section{Publisher's Note}

Springer Nature remains neutral with regard to jurisdictional claims in published maps and institutional affiliations.
Ready to submit your research? Choose BMC and benefit from:

- fast, convenient online submission

- thorough peer review by experienced researchers in your field

- rapid publication on acceptance

- support for research data, including large and complex data types

- gold Open Access which fosters wider collaboration and increased citations

- maximum visibility for your research: over $100 \mathrm{M}$ website views per year

At BMC, research is always in progress.

Learn more biomedcentral.com/submissions 